\title{
QUADRATIC HARNESSES FROM GENERALIZED BETA INTEGRALS
}

\author{
WŁODEK BRYC
}

\begin{abstract}
We use generalized beta integrals to construct examples of Markov processes with linear regressions, and quadratic second conditional moments.
\end{abstract}

\section{INTRODUCTION}

1.1. Quadratic harnesses. In [BMW07] the authors consider square-integrable stochastic processes on $(0, \infty)$ such that for all $t, s>0$,

$$
\mathbb{E}\left(X_{t}\right)=0, \mathbb{E}\left(X_{t} X_{s}\right)=\min \{t, s\},
$$

$\mathbb{E}\left(X_{t} \mid \mathcal{F}_{s, u}\right)$ is a linear function of $X_{s}, X_{u}$, and $\operatorname{Var}\left[X_{t} \mid \mathcal{F}_{s, u}\right]$ is a quadratic function of $X_{s}, X_{u}$. Here, $\mathcal{F}_{s, u}$ is the two-sided $\sigma$-field generated by $\left\{X_{r}: r \in(0, s] \cup[u, \infty)\right\}$. Then for all $s<t<u$, (1.1) implies that

$$
\mathbb{E}\left(X_{t} \mid \mathcal{F}_{s, u}\right)=\frac{u-t}{u-s} X_{s}+\frac{t-s}{u-s} X_{u},
$$

which is sometimes referred to as a harness condition, see [MY05. While there are numerous examples of harnesses that include all integrable Lévy processes ([JP88, (2.8)]), the assumption of quadratic conditional variance is more restrictive, see [Wes93]. Under certain assumptions, [BMW07, Theorem 2.2] asserts that there exist numerical constants $\eta, \theta \in \mathbb{R} \sigma, \tau>0$ and $\gamma \in[-1,1+2 \sqrt{\sigma \tau}]$ such that for all $s<t<u$,

$$
\begin{aligned}
\operatorname{Var}\left[X_{t} \mid \mathcal{F}_{s, u}\right]= & \frac{(u-t)(t-s)}{u(1+\sigma s)+\tau-\gamma s}\left(1+\eta \frac{u X_{s}-s X_{u}}{u-s}+\theta \frac{X_{u}-X_{s}}{u-s}\right. \\
& \left.\quad+\sigma \frac{\left(u X_{s}-s X_{u}\right)^{2}}{(u-s)^{2}}+\tau \frac{\left(X_{u}-X_{s}\right)^{2}}{(u-s)^{2}}-(1-\gamma) \frac{\left(X_{u}-X_{s}\right)\left(u X_{s}-s X_{u}\right)}{(u-s)^{2}}\right) .
\end{aligned}
$$

We will say that a square-integrable stochastic process $\left(X_{t}\right)_{t \in T}$ is a quadratic harness on $T$ with parameters $(\eta, \theta, \sigma, \tau, \gamma)$, if it satisfies (1.1), (1.2) and (1.3) on an open interval $T \subset(0, \infty)$.

Our goal is to construct examples of Markov quadratic harnesses with $\gamma=1-2 \sqrt{\sigma \tau}$. In BMW07, Proposition 4.4], these were called "classical quadratic harnesses. The construction follows [BW10, Section 2] who construct quadratic harnesses with $\gamma<1-2 \sqrt{\sigma \tau}$ from the Askey-Wilson integral. Here we use instead some of the generalized Beta integrals from [Ask89.

The paper is organized into sections based on the number of parameters in the generalized beta integrals. In particular, in Section 4 we exhibit explicit transition probabilities for the bridges of the hyperbolic secant process, and for completeness in Section 5 we re-analyze the Dirichlet process.

Date: Created: October 27, 2009.

Printed: October 30, 2018 file: Wilson09v4.tex.

2000 Mathematics Subject Classification. 60J25.

Key words and phrases. Quadratic conditional moments, generalized beta integrals, harnesses. 
1.2. Conversion to the standard form. In this section we recall a procedure that we use to transform Markov processes with linear regressions and quadratic conditional variances into the quadratic harnesses. The following is a specification of [BW09, Theorem 1.1] that fits our needs.

Proposition 1.1. Suppose $\left(Y_{t}\right)$ is a (real-valued) Markov process on an open interval $T \subset \mathbb{R}$ such that

(1) $\mathbb{E}\left(Y_{t}\right)=\alpha+\beta t$ for some real $\alpha, \beta$.

(2) For $s<t$ in $T, \operatorname{Cov}\left(Y_{s}, Y_{t}\right)=M^{2}(\psi+s)(\delta+\varepsilon t)$, where $M^{2}(\psi+t)(\delta+\varepsilon t)>0$ on the entire interval $T$, and that $\delta-\varepsilon \psi>0$.

(3) For $s<t<u$,

$$
\operatorname{Var}\left(Y_{t} \mid Y_{s}, Y_{u}\right)=F_{t, s, u}\left(\chi_{0}+\eta_{0} \frac{u Y_{s}-s Y_{u}}{u-s}+\theta_{0} \frac{Y_{u}-Y_{s}}{u-s}+\frac{\left(Y_{u}-Y_{s}\right)^{2}}{(u-s)^{2}}\right),
$$

where $F_{t, s, u}$ is non-random and $\chi_{0}, \theta_{0}, \eta_{0} \in \mathbb{R}$ are such that $\chi:=\chi_{0}+\alpha \eta_{0}+\beta \theta_{0}+\beta^{2}>0$. Denote $\widetilde{Y}_{t}=Y_{t}-\mathbb{E}\left(Y_{t}\right)$. Then there are two affine functions $\ell(t)=\frac{t \delta-\psi}{M(\delta-\epsilon \psi)}$ and $m(t)=\frac{1-t \epsilon}{M(\delta-\epsilon \psi)}$ and an open interval $T^{\prime} \subset(0, \infty)$ such that $X_{t}:=m(t) \widetilde{Y}_{\ell(t) / m(t)}$ defines a process $\left(X_{t}\right)$ on $T^{\prime}$ such that (1.1) holds and (1.3) holds with parameters

$$
\begin{aligned}
\eta & =M\left(\delta \eta_{0}+\epsilon\left(2 \beta+\theta_{0}\right)\right) / \chi \\
\theta & =M\left(2 \beta+\psi \eta_{0}+\theta_{0}\right) / \chi, \\
\sigma & =M^{2} \varepsilon^{2} / \chi, \\
\tau & =M^{2} / \chi \\
\gamma & =1+2 \varepsilon \sqrt{\sigma \tau} .
\end{aligned}
$$

Proof. This is [BW09, Theorem 1.1] specialized to $\chi=\chi_{0}, \eta=\eta_{0}, \theta=\theta_{0}, \sigma=0, \tau=1, \rho=0$, $a=M, b=M \psi, c=M \epsilon, d=M \delta$.

Remark 1.1. We will apply this only to $\varepsilon=0, \pm 1$, and $\chi_{0}, \theta_{0}, \eta_{0} \in\{0,1\}$.

Remark 1.2. For $\varepsilon \leq 0$, we see that $\gamma \leq 1$ and $\eta \sqrt{\tau}+\theta \sqrt{\sigma}=M^{2}(\delta-\varepsilon \psi) \eta_{0} / \chi^{2}$ has the same sign as $\eta_{0}$.

Remark 1.3. The time domain $T^{\prime}$ is the image of $T$ under the Möbius transformation $t \mapsto$ $(t+\psi) /(\varepsilon t+\delta)$.

Two related transformations are sometimes useful to keep in mind, as they take care of some additional non-uniqueness in the final form of (1.3). Firstly, if $\left(X_{t}\right)$ is a quadratic harness with parameters $(\eta, \theta, \sigma, \tau, \gamma)$ then $\left(a X_{t / a^{2}}\right)$ is a quadratic harness with parameters $\left(\eta / a, a \theta, \sigma / a^{2}, a^{2} \tau, \gamma\right)$. In particular, if $\sigma=0$ and $\tau>0$, then without loss of generality we may take $\tau=1$. And if $\sigma, \tau>0$ then without loss of generality we may take $\sigma=\tau$. (So our constructions will lead to these two cases only.)

Secondly, time inversion $\left(t X_{1 / t}\right)$ converts a quadratic harness with parameters $(\eta, \theta, \sigma, \tau, \gamma)$ into a quadratic harness with parameters $(\theta, \eta, \tau, \sigma, \gamma)$, i.e. it swaps the entries within pairs $(\eta, \theta)$ and $(\sigma, \tau)$. In particular, time inversion maps a quadratic harness with $\sigma=0, \tau=1$ into a quadratic harness with $\sigma=1, \tau=0$. Similarly, it maps a quadratic harness with parameters $\sigma=\tau$ and $\eta^{2}<4 \sigma, \theta^{2} \geq 4 \sigma$ into a quadratic harness with parameters $\sigma=\tau$ and $\eta^{2} \geq 4 \sigma, \theta<4 \sigma$.

\section{Four-PARAmeter Beta Integral}

This section contains the construction of Markov processes based on the four-parameter beta integral [Ask89, (8.i)]. After a transformation, these processes become quadratic harnesses with 
arbitrary $\sigma=\tau \in(0,1), \gamma=1-2 \sqrt{\sigma \tau}$, and with $\eta, \theta$ such that $\sqrt{\tau} \eta+\sqrt{\sigma} \theta \neq 0$; parameters $\eta, \theta$ will be required to satisfy also some additional restrictions, of which $\eta \theta \geq 0$ suffices for all constructions to go through. Since the main steps will be repeated several times, first with three parameters to cover the case $\sigma=0$, and then with two parameters to cover the case $\sqrt{\tau} \eta+\sqrt{\sigma} \theta=0$, we give here more details so that we can suppress them in the subsequent iterations.

The construction starts with four complex numbers $a_{1}, a_{2}, a_{3}, a_{4}$ with strictly positive real parts. The generalized beta integral dB72, Wil80] after changing the variable to $\sqrt{x}$ is

$$
\int_{0}^{\infty} \frac{\prod_{j=1}^{4}\left(\Gamma\left(a_{j}+i \sqrt{x}\right) \Gamma\left(a_{j}-i \sqrt{x}\right)\right)}{\sqrt{x}|\Gamma(2 i \sqrt{x})|^{2}} d x=\frac{4 \pi \prod_{1 \leq k<j \leq 4} \Gamma\left(a_{k}+a_{j}\right)}{\Gamma\left(a_{1}+a_{2}+a_{3}+a_{4}\right)} .
$$

Denote

$$
K(a, b, c, d)=\frac{\Gamma(a+b+c+d)}{4 \pi \Gamma(a+b) \Gamma(a+c) \Gamma(b+c) \Gamma(a+d) \Gamma(b+d) \Gamma(c+d)} .
$$

If $a, b, c, d$ are positive real numbers, or come as one or two conjugate pairs with positive real parts, identity (2.1) implies that the following function of $x>0$ becomes a four-parameter probability density function on $(0, \infty)$ :

$$
f(x ; a, b, c, d)=K(a, b, c, d) \frac{|\Gamma(a+i \sqrt{x}) \Gamma(b+i \sqrt{x}) \Gamma(c+i \sqrt{x}) \Gamma(d+i \sqrt{x})|^{2}}{\sqrt{x}|\Gamma(2 i \sqrt{x})|^{2}} .
$$

Proposition 2.1. If a random variable $X$ has density $f(x ; a, b, c, d)$ then

$$
\mathbb{E}(X)=\frac{a b c+a b d+a c d+b c d}{a+b+c+d}
$$

and

$$
\operatorname{Var}(X)=\frac{(a+b)(a+c)(b+c)(a+d)(b+d)(c+d)}{(a+b+c+d)^{2}(a+b+c+d+1)}
$$

Proof. The formulas can be read out from the first two orthogonal polynomials [KS98, (1.1.4)], but they also follow easily from the formulas

$$
\mathbb{E}\left(a^{2}+X\right)=\mathbb{E}((a+i \sqrt{X})(a-i \sqrt{X}))=\frac{K(a, b, c, d)}{K(a+1, b, c, d)}
$$

and

$$
a^{2} b^{2}+\left(a^{2}+b^{2}\right) \mathbb{E}(X)+\mathbb{E}\left(X^{2}\right)=\mathbb{E}\left(\left(a^{2}+X\right)\left(b^{2}+X\right)\right)=\frac{K(a, b, c, d)}{K(a+1, b+1, c, d)} .
$$

Now using (2.2) and $s \Gamma(s)=\Gamma(s+1)$, we get (2.4), and after a calculation we get (2.5) .

Next, we prove a "convolution formula" which will be used to verify the Chapman-Kolmogorov equations.

Proposition 2.2. If $m>0$ then

$$
f(y ; a, b, c+m, d+m)=\int_{0}^{\infty} f(y ; a, b, m+i \sqrt{x}, m-i \sqrt{x}) f(x ; a+m, b+m, c, d) d x .
$$

Proof. Re-arranging the factors in (2.3), we have

$$
\frac{f(x, a+m, b+m, c, d) f(y, a, b, m+i \sqrt{x}, m-i \sqrt{x})}{f(y, a, b, c+m, d+m)}=f(x, m+i \sqrt{y}, m-i \sqrt{y}, c, d) .
$$

Formula (2.6) now follows, as $\int_{0}^{\infty} f(x ; \mu+i \sqrt{y}, \mu-i \sqrt{y}, c, d) d x=1$. 
We remark that (2.7) is an analog of [Jam74, (b3)] and will serve similar purposes. Related formulas will appear again as (3.3), (4.5), (4.14), and (5.3).

2.1. The auxiliary Markov process. We now define a family of Markov processes $\left(Y_{t}\right)_{t \in T}$, parameterized by $A, B, C, D$ that are either all real and positive or come as one or two complex conjugate pairs $A=\bar{B}$ or $C=\bar{D}$, with positive real parts. Without loss of generality we may assume that $\Re(A) \leq \Re(B)$ and $\Re(C) \leq \Re(D)$.

As the time domain for Markov process $\left(Y_{t}\right)$ we take the open interval $T=(-\Re(C), \Re(A))$, and as the state space we take $(0, \infty)$. We define the univariate distribution of $Y_{t}$ by the density

$$
f_{t}(x)=f(x ; A-t, B-t, C+t, D+t) .
$$

For $s<t$, we define the transition probability $\mathcal{L}\left(Y_{t} \mid Y_{s}=x\right)$ by the density

$$
f_{s, t}(y \mid x)=f(y ; A-t, B-t, t-s+i \sqrt{x}, t-s-i \sqrt{x}) .
$$

It remains to verify that the above definitions are consistent.

Proposition 2.3. Formulas (2.8) and (2.9) determine a Markov process $\left(Y_{t}\right)_{t \in T}$. Furthermore, $\mathbb{E}\left(Y_{0}\right)=(A B C+A B D+A C D+B C D) /(A+B+C+D)$ by (2.4) and

$$
\mathbb{E}\left(Y_{t}\right)=\mathbb{E}\left(Y_{0}\right)+2 \frac{A B-C D}{A+B+C+D} t-t^{2}
$$

For $s \leq t$ in $T$,

$$
\operatorname{Cov}\left(Y_{s}, Y_{t}\right)=M^{2}(C+D+2 s)(A+B-2 t)
$$

where

$$
M^{2}=\frac{(A+C)(B+C)(A+D)(B+D)}{(A+B+C+D)^{2}(A+B+C+D+1)}>0 .
$$

In view of (2.10), the conditional moments simplify when we express them in terms of

$$
\widetilde{Y}_{t}=Y_{t / 2}+t^{2} / 4,-2 \Re(C)<t<2 \Re(A),
$$

with linear mean $\mathbb{E}\left(\widetilde{Y}_{t}\right)=\alpha+\beta t$ and the covariance $\operatorname{Cov}\left(\widetilde{Y}_{s}, \widetilde{Y}_{t}\right)=M^{2}(C+D+s)(A+B-t)$ for $s \leq t$. The one-sided conditional moments $s \leq t$ are:

$$
\begin{gathered}
\mathbb{E}\left(\widetilde{Y}_{t} \mid \widetilde{Y}_{s}\right)=\frac{(A+B-t)}{A+B-s} \widetilde{Y}_{s}+\frac{A B(t-s)}{A+B-s} \\
\operatorname{Var}\left(\widetilde{Y}_{t} \mid \widetilde{Y}_{s}\right)=\frac{(A+B-t)(t-s)\left(A^{2}-s A+\widetilde{Y}_{s}\right)\left(B^{2}-s B+\widetilde{Y}_{s}\right)}{(A+B-s)^{2}(A+B-s+1)} .
\end{gathered}
$$

For $s<t<u$ in $T$,

$$
\begin{gathered}
\mathbb{E}\left(\widetilde{Y}_{t} \mid \widetilde{Y}_{s}, \widetilde{Y}_{u}\right)=\frac{(u-t) \tilde{Y}_{s}+(t-s) \tilde{Y}_{u}}{u-s} \\
\operatorname{Var}\left(\widetilde{Y}_{t} \mid \widetilde{Y}_{s}, \widetilde{Y}_{u}\right)=\frac{(u-t)(t-s)}{u-s+1}\left(\frac{\left(\widetilde{Y}_{u}-\widetilde{Y}_{s}\right)^{2}}{(u-s)^{2}}+\frac{u \widetilde{Y}_{s}-s \widetilde{Y}_{u}}{u-s}\right) .
\end{gathered}
$$


Proof. To verify Chapman-Kolmogorov equations we first use (2.6) with $m=t-s, a=A-t$, $b=B-t, c=C+t, d=D+t$. This gives

$$
f_{t}(y)=\int_{0}^{\infty} f_{s, t}(y \mid x) f_{s}(x) d x .
$$

Next we use (2.6) with $m=u-t, a=A-u, b=B-u, c=t-s+i \sqrt{x}, d=t-s-i \sqrt{x}$ to verify the Chapman-Kolmogorov equations for the transition probabilities,

$$
f_{s, u}(z \mid x)=\int_{0}^{\infty} f_{s, t}(y \mid x) f_{t, u}(z \mid y) d y
$$

Formula (2.7) can be now reinterpreted as the formula for the conditional distribution $\mathcal{L}\left(Y_{t} \mid Y_{s}=\right.$ $\left.x, Y_{u}=z\right)$, given by the density

$$
g(y \mid x, z)=\frac{f_{t, u}(z \mid y) f_{s, t}(y \mid x)}{f_{s, u}(z \mid x)}=f(y ; u-t+i \sqrt{z}, u-t-i \sqrt{z}, t-s+i \sqrt{x}, t-s-i \sqrt{x}) .
$$

Since this is again expressed in terms of the same density (2.3), the formulas for the conditional mean and conditional variance are recalculated from Proposition 2.1. Finally, we use (2.10), and

$$
\operatorname{Var}\left(Y_{t}\right)=M^{2}(A+B-2 t)(C+D+2 t),
$$

which is calculated from (2.5) , and (2.14), to compute $\mathbb{E}\left(Y_{s} Y_{t}\right)$ and we get (2.11).

Corollary 2.4. $\left(\widetilde{Y}_{t}\right)$ can be transformed into a quadratic harness with covariance (1.1) and the conditional variance (1.3) with parameters

$$
\begin{aligned}
\eta+\theta & =\frac{(A+B+C+D)^{2}}{\sqrt{(A+C)(B+C)(A+D)(B+D)(A+B+C+D+1)}}, \\
\theta-\eta & =\frac{(C-D)^{2}-(A-B)^{2}}{\sqrt{(A+C)(B+C)(A+D)(B+D)(A+B+C+D+1)}}, \\
\sigma=\tau & =\frac{1}{A+B+C+D+1},
\end{aligned}
$$

and $\gamma=1-2 \sqrt{\sigma \tau}=(A+B+C+D-1) /(A+B+C+D+1)$.

Proof. We apply Proposition 1.1 with parameters

$$
\alpha=\frac{A B C+A D C+B D C+A B D}{A+B+C+D}, \beta=\frac{A B-C D}{A+B+C+D}, \epsilon=-1, \psi=C+D, \delta=A+B .
$$

The only non-zero parameters in (1.4) are $\eta_{0}=\tau_{0}=1$.

Remark 2.1. The quadratic harness is defined on the interval

$$
T^{\prime}=\left(\frac{C+D-2 \Re(C)}{A+B+2 \Re(C)}, \frac{C+D+2 \Re(A)}{A+B-2 \Re(A)}\right) .
$$

In particular, $T^{\prime}=(0, \infty)$ if $A=\bar{B}$ and $C=\bar{D}$. It is plausible that by allowing transition probabilities and univariate laws with discrete components, this interval could be extended to $(0, \infty)$ in all cases when $\Re(A+B)>0$ and $\Re(C+D)>0$. 
2.2. The admissible range of $\eta, \theta$. In this section we study which collections of parameters correspond to quadratic harnesses from the previous construction. Given $\sigma=\tau, \gamma=1-2 \sigma$ and $\eta, \theta$ such that $\eta+\theta \neq 0$, without loss of generality we may assume that $\eta+\theta>0$. For if we can find a quadratic harness $\left(X_{t}\right)$ for one such set of parameters, then $\left(-X_{t}\right)$ is a quadratic harness on the same time domain, with the same $\sigma, \tau, \gamma$, but with $-\eta,-\theta$ instead of $\eta, \theta$.

Once we restrict ourselves to the case $\eta+\theta>0$, we want to know for which $\eta, \theta, \sigma=\tau, \gamma=1-2 \sigma$ we can find $A, B, C, D$ that satisfy the equations from Corollary 2.4 and satisfy the constraints for the construction of the Markov process $\left(Y_{t}\right)$. We will see that we can always find such $A, B, C, D$ if either $\eta \theta \geq 0$ (which under the assumption $\eta+\theta>0$, is equivalent to $\eta \geq 0, \theta>0$ or vice versa) or when the sign of $\eta \theta$ is arbitrary but $\eta^{2}<4 \sigma$ and $\theta^{2}<4 \tau$.

To proceed, we rewrite the equations from Corollary 2.4 in equivalent form:

$$
\begin{aligned}
A+B+C+D & =(1-\sigma) / \sigma, \\
(A+C)(B+C)(A+D)(B+D) & =\frac{(1-\sigma)^{4}}{(\eta+\theta)^{2} \sigma^{3}} \\
(C-D)^{2}-(A-B)^{2} & =\frac{(\theta-\eta)(1-\sigma)^{2}}{(\eta+\theta) \sigma^{2}} .
\end{aligned}
$$

2.2.1. Hyperbolic case. We first show that quadratic harnesses exist for any $\eta, \theta$ such that $\eta+\theta>$ $0, \eta^{2}<4 \sigma$ and $\theta^{2}<4 \sigma$. This is because in this case the system of equations (2.25+2.27) is solved by two conjugate pairs $A=\bar{B}$ and $C=\bar{D}$ with $A, C$ given by

$$
A=\Re(A)+\frac{i(1-\sigma) \sqrt{4 \sigma-\eta^{2}}}{2(\eta+\theta) \sigma}, C=\frac{1-\sigma}{2 \sigma}-\Re(A)+\frac{i(1-\sigma) \sqrt{4 \sigma-\theta^{2}}}{2(\eta+\theta) \sigma}
$$

with arbitrary $0<\Re(A)<\frac{1-\sigma}{2 \sigma}$.

The apparent non-uniqueness in this solution and in others is in fact illusory, as it corresponds to the translation of the time domain $T$. This translation does not affect neither the transition probabilities of the final quadratic harness, nor the final time domain, which by Remark 2.1 is $T^{\prime}=(0, \infty)$.

2.2.2. Next we go over the remaining choices of pairs $(\eta, \theta)$, and confirm that in each case we can always find a quadratic harness when $\eta \theta \geq 0$.

We first consider $\eta, \theta$ such that $\eta+\theta>0, \eta^{2}<4 \sigma$ and $\theta^{2} \geq 4 \sigma$. Then quadratic harnesses exist if $4 \sigma+\eta^{2}+2 \eta \theta>0$. Indeed, in this case the system of equations (2.25) 2.27) is solved with one conjugate pair $A=\bar{B}$. The solutions are

$$
\begin{gathered}
A=\Re(A)+\frac{i(1-\sigma) \sqrt{4 \sigma-\eta^{2}}}{2(\eta+\theta) \sigma}, \\
C=\frac{\left(\eta+\theta-\sqrt{\theta^{2}-4 \sigma}\right)(1-\sigma)}{2(\eta+\theta) \sigma}-\Re(A), \\
D=\frac{\left(\eta+\theta+\sqrt{\theta^{2}-4 \sigma}\right)(1-\sigma)}{2(\eta+\theta) \sigma}-\Re(A) .
\end{gathered}
$$

The restriction $4 \sigma+\eta^{2}+2 \eta \theta>0$ guarantees that $\theta^{2}-4 \sigma<(\theta+\eta)^{2}$ so one can find $\Re(A)>0$ such that $C>0$; then $D>0$ follows automatically.

The restriction $4 \sigma+\eta^{2}+2 \eta \theta>0$ holds, in particular, if $\eta \geq 0$, as then $4 \sigma+\eta^{2}+2 \eta \theta>$ $2 \eta^{2}+2 \eta \theta=2 \eta(\eta+\theta) \geq 0$. 
We remark that the left endpoint of the time domain $T^{\prime}$ here is 0 , see Remark 2.1. This is of interest, since for such domains the one-sided conditional moments (2.14) and (2.15) imply uniqueness of the quadratic harness.

Finally, if $\eta+\theta>0$ are such that $\eta^{2} \geq 4 \sigma$ and $\theta^{2} \geq 4 \sigma$, then under the condition $\eta+\theta>$ $\sqrt{\eta^{2}-4 \sigma}+\sqrt{\theta^{2}-4 \sigma}$ one can choose a small enough $A>0$ such that

$$
\begin{gathered}
B=A+\frac{\sqrt{\eta^{2}-4 \sigma}(1-\sigma)}{(\eta+\theta) \sigma}, \\
C=\frac{\left(\eta+\theta-\sqrt{\eta^{2}-4 \sigma}-\sqrt{\theta^{2}-4 \sigma}\right)(1-\sigma)}{2(\eta+\theta) \sigma}-A, \\
D=\frac{\left(\eta+\theta-\sqrt{\eta^{2}-4 \sigma}+\sqrt{\theta^{2}-4 \sigma}\right)(1-\sigma)}{2(\eta+\theta) \sigma}-A,
\end{gathered}
$$

are all positive. In particular, if $\eta, \theta>0$ then $\eta>\sqrt{\eta^{2}-4 \sigma}$ and $\theta>\sqrt{\theta^{2}-4 \sigma}$ so the above solution will indeed give us a quadratic harness on a finite interval $T^{\prime}$.

\section{Three PARAMETER BetA INTEGRAL}

For $a>0$ and $b, c$ real positive or a complex conjugate pair with positive real part, define the following density on $[0, \infty)$ : (See [Ask89, (7.i)] or [KS98, Section 1.3])

$$
g(x ; a, b, c)=\frac{|\Gamma(a+i \sqrt{x}) \Gamma(b+i \sqrt{x}) \Gamma(c+i \sqrt{x})|^{2}}{4 \pi \Gamma(a+b) \Gamma(a+c) \Gamma(b+c) \sqrt{x}|\Gamma(2 i \sqrt{x})|^{2}} .
$$

As previously, it is straightforward to use properties of the gamma function to get formulas for the mean $\mu$ and the variance $\sigma^{2}$,

$$
\mu=a b+a c+b c, \sigma^{2}=(a+b)(a+c)(b+c) .
$$

The relevant version of (2.7) is

$$
\frac{g(x ; a+m, b, c) g(y ; a, m+i \sqrt{x}, m-i \sqrt{x})}{g(y ; a, b+m, c+m)}=f(x, m+i \sqrt{y}, m-i \sqrt{y}, b, c),
$$

where $x, y, m>0$.

Let $A \in \mathbb{R}$ and let $B, C$ be either real or a complex conjugate pair, and without loss of generality we assume that in the real case $B \geq C$. Suppose in addition that $A+\Re(C)>0$ so that $T=(-\Re(C), A)$ is non-empty. Then from (3.3) we get again a Markov process $\left(Y_{t}\right)_{t \in T}$ with univariate distributions on the state space $(0, \infty)$ defined by the densities $g(x ; A-t, B+$ $t, C+t)$, with transition probabilities defined for $s<t$ in $T$ and $x, y>0$ by the densities $g(y ; A-t, t-s-i \sqrt{x}, t-s+i \sqrt{x})$, and whose two-sided conditional laws are again given by Wilson's density (2.20). In particular, after we make substitution (2.13) formulas (2.16) and (2.17) for the two-sided conditional mean and variance hold.

As previously, parameters $A, B, C$ affect only the mean and the covariance of $\left(Y_{t}\right)$ :

$$
\mathbb{E}\left(Y_{t}\right)=-t^{2}+2 A t+A B+A C+B C, \operatorname{Var}\left(Y_{t}\right)=(A+B)(A+C)(B+C+2 t) .
$$

Passing to the centered process (2.13), the one-sided conditional moments are:

$$
\mathbb{E}\left(\tilde{Y}_{t} \mid \tilde{Y}_{s}\right)=A(t-s)+\tilde{Y}_{s}, \operatorname{Var}\left(\tilde{Y}_{t} \mid \tilde{Y}_{s}\right)=(t-s)\left(A^{2}-s A+\tilde{Y}_{s}\right) .
$$

In particular, the above formula for $\mathbb{E}\left(\widetilde{Y}_{t} \mid \widetilde{Y}_{s}\right)$ gives

$$
\operatorname{Cov}\left(\widetilde{Y}_{s}, \widetilde{Y}_{t}\right)=(A+B)(A+C)(B+C+\min \{t, s\}) .
$$


Then the transformation from Proposition 1.1 takes a particularly simple form. Markov process

$$
X_{t}=\frac{\tilde{Y}_{t-B-C}-\mathbb{E}\left(\tilde{Y}_{t-B-C}\right)}{\sqrt{(A+B)(A+C)}}
$$

is a quadratic harness with parameters

$$
\begin{aligned}
\eta & =\frac{1}{\sqrt{(A+B)(A+C)}} \\
\theta & =\frac{2 A+B+C}{\sqrt{(A+B)(A+C)}} \\
\sigma & =0 \\
\tau & =1 .
\end{aligned}
$$

This gives us a family of quadratic harnesses arbitrary positive values for parameters $\eta, \theta$, with $\tau=1, \sigma=0$. Other values of parameters are now produced by routine transformations that were mentioned in the introduction. To swap the roles of $\sigma, \tau$ one uses time inversion $\left(t X_{1 / t}\right)$. Taking $\left(-X_{t}\right)$ we get arbitrary negative values of $\eta, \theta$, covering all possible non-zero values of the same sign $(\eta \theta>0)$. Finally, transformation $\left(X_{\alpha t} / \sqrt{\alpha}\right)$ produces arbitrary positive values for parameter $\tau$.

Remark 3.1. The quadratic harness is defined on

$$
T^{\prime}=(\Re(C-B), \infty) .
$$

In particular, $T^{\prime}=(0, \infty)$ if $B=\bar{C}$. It would be interesting to see if the construction could be modified to yield $T^{\prime}=(0, \infty)$ also for real $B \neq C$.

Remark 3.2. Formula (3.3) indicates that bridges of the three-parameter quadratic harnesses with $\sigma=0$ are the (transformations of) four-parameter quadratic harnesses from Corollary 2.4. It would be interesting to see if this holds true also in the cases without densities.

\section{TWO-PARAMETER BETA INTEGRAL}

According to [Ask89, (5.i)], see also [KS98, Section 1.4], the following is a probability density on $\mathbb{R}$ when $c=\bar{a}, d=\bar{b}$ have positive real part.

$$
\varphi(x ; a, b, c, d)=\frac{\Gamma(a+b+c+d) \Gamma(a+i x) \Gamma(b+i x) \Gamma(c-i x) \Gamma(d-i x)}{2 \pi \Gamma(a+c) \Gamma(b+c) \Gamma(a+d) \Gamma(b+d)} .
$$

The analog of Proposition 2.1 is

Proposition 4.1. If a random variable $X \in \mathbb{R}$ has density $\varphi(x ; a, b, c, d)$, then

$$
\begin{gathered}
\mathbb{E}(X)=-\frac{\Re(a) \Im(b)+\Re(b) \Im(a)}{\Re(a+b)}, \\
\operatorname{Var}(X)=\frac{\Re(a) \Re(b)\left((\Re(a+b))^{2}+(\Im(a-b))^{2}\right)}{(\Re(a+b))^{2}(2 \Re(a+b)+1)} .
\end{gathered}
$$


Proof. Denote by $K(a, b, c, d)=\frac{\Gamma(a+b+c+d)}{2 \pi \Gamma(a+c) \Gamma(b+c) \Gamma(a+d) \Gamma(b+d)}$ the normalizing constant in (4.1). Then

$$
\begin{aligned}
& \int_{-\infty}^{\infty} x \varphi(x ; a, b, c, d) d x \\
= & \frac{1}{i(c+b-a-d)}\left(\int_{-\infty}^{\infty}((a+i x)(c-i x)-(b+i x)(d-i x)+b d-a c) \varphi(x ; a, b, c, d) d x\right) \\
= & \frac{K(a, b, c, d)}{i(c+b-a-d)}\left(\frac{1}{K(a+1, b, c+1, d)}-\frac{1}{K(a, b+1, c, d+1)}+b d-a c\right)=\frac{i(a b-c d)}{a+b+c+d} .
\end{aligned}
$$

Substituting $a=\Re(a)+i \Im(a), b=\Re(b)+i \Im(b), c=\Re(a)-i \Im(a), d=\Re(b)-i \Im(b)$ we get (4.2).

The variance comes from a similar calculation:

$$
\begin{aligned}
\mathbb{E}\left(X^{2}\right)-(\mathbb{E}(X))^{2}=K(a+1, b, c+1, d)-(c-a) \mathbb{E}(X) & -a c-(\mathbb{E}(X))^{2} \\
& =\frac{(a+c)(b+c)(a+d)(b+d)}{(a+b+c+d)^{2}(a+b+c+d+1)} .
\end{aligned}
$$

The analog of Proposition 2.2 is based on the identity

$$
\frac{\varphi(y ; a, m-i x, \bar{a}, m+i x) \varphi(x ; a+m, b, \bar{a}+m, \bar{b})}{\varphi(y ; a, b+m, \bar{a}, \bar{b}+m)}=\varphi(x ; b, m-i y, \bar{b}, m+i y) .
$$

Thus, given complex parameters $A, B$, such that $\Re(A+B)>0$, let $T=(-\Re(B), \Re(A))$. For $s<t$ in $T$, the univariate densities on $\mathbb{R}$

$$
f_{t}(x)=\varphi(x, A-t, B+t, \bar{A}-t, \bar{B}+t),
$$

and the transition probabilities

$$
f_{s, t}(y \mid x)=\varphi(x, A-t, t-s-i x, \bar{A}-t, t-s+i x),
$$

satisfy the Chapman-Kolmogorov equations (2.18) and (2.19). Let $\left(Y_{t}\right)_{t \in T}$ denote the corresponding Markov process. Then from (4.2) and (4.3) we get

$$
\mathbb{E}\left(Y_{t}\right)=\frac{\Im(B-A)}{\Re(A+B)} t-\frac{\Re(A) \Im(B)+\Im(A) \Re(B)}{\Re(A+B)}, \operatorname{Var}\left(Y_{t}\right)=M^{2}(\Re(A)-t)(\Re(B)+t),
$$

where

$$
M^{2}=\frac{(\Im(A-B))^{2}+(\Re(A+B))^{2}}{(\Re(A+B))^{2}(2 \Re(A+B)+1)} .
$$

Since (4.2) also gives

$$
\mathbb{E}\left(Y_{t} \mid Y_{s}\right)=\frac{\Re(A)-t}{\Re(A)-s} Y_{s}-\frac{\Im(A)(t-s)}{\Re(A)-s}
$$

for $s<t$, from (4.8) we further calculate

$$
\operatorname{Cov}\left(Y_{s}, Y_{t}\right)=M^{2}(\Re(A)-t)(\Re(B)+s) .
$$

Next we compute conditional moments. For $s<t<u$ in, the two-sided conditional density of $\mathcal{L}\left(Y_{t} \mid Y_{s}=x, Y_{u}=z\right)$ is given by

$$
g(y \mid x, z)=\varphi(y ; t-s-i x, u-t-i z, t-s+i x, u-t+i z) .
$$

So from (4.2),

$$
\mathbb{E}\left(Y_{t} \mid Y_{s}, Y_{u}\right)=\frac{(u-t) Y_{s}+(t-s) Y_{u}}{u-s}
$$


and from (4.3) we get

$$
\operatorname{Var}\left(Y_{t} \mid Y_{s}, Y_{u}\right)=\frac{(t-s)(u-t)}{(2 u-2 s+1)}\left(1+\frac{\left(Y_{u}-Y_{s}\right)^{2}}{(u-s)^{2}}\right) .
$$

From Proposition 1.1 applied with $\chi_{0}=1, \alpha=-\frac{\Im(B) \Re(A)+\Im(A) \Re(B)}{\Re(A+B)}, \beta=\frac{\Im(B-A)}{\Re(A+B)}, \eta_{0}=0$, $\theta_{0}=0, \epsilon=-1, \psi=\Re(B), \delta=\Re(A), M=\frac{\sqrt{(\Im(A-B))^{2}+(\Re(A+B))^{2}}}{\Re(A+B) \sqrt{2 \Re(A+B)+1}}$, we get

$$
\begin{gathered}
\sigma=\tau=\frac{1}{2 \Re(A+B)+1}, \\
\eta=-\theta=\frac{2(\Im(A-B))}{\sqrt{(2 \Re(A+B)+1)\left((\Im(A-B))^{2}+(\Re(A+B))^{2}\right)}} .
\end{gathered}
$$

From the first equation, we see that $\Re(A+B)=\frac{1-\sigma}{2 \sigma}$. The second equation determines $\Im(A-B)$ as a real number iff $\theta^{2}<4 \tau$. This proves the following.

Proposition 4.2. For every $\sigma \in(0,1)$ and $\eta \in(-2 \sqrt{\sigma}, 2 \sqrt{\sigma})$, there is a quadratic harness on $(0, \infty)$ with parameters $\eta, \theta=-\eta, \sigma, \tau=\sigma, \gamma=1-2 \sigma$.

4.1. Bridges of the hyperbolic secant process. Bridges of all Meixner processes are described in [BW09, Proposition 4.2 and Remark 4.1]. According to these results, bridges of Meixner processes are quadratic harnesses with $\eta \sqrt{\tau}+\theta \sqrt{\sigma}=0$. When $\sigma \tau>0$, then depending on the sign of $\theta^{2}-4 \tau$, such processes arise as bridges of negative binomial, gamma, or the hyperbolic secant process. In [BW09] the bridges of hyperbolic secant process were not described explicitly, so we identify their transition probabilities here.

The following integral is due to Meixner [Mei34, page 13], and is listed as [Ask89, (4.i)]:

$$
\int_{-\infty}^{\infty}|\Gamma(a+i x)|^{2} e^{\beta x} d x=\frac{2 \pi \Gamma(2 a)}{\left(2 \cos \frac{\beta}{2}\right)^{2 a}} .
$$

The integral is well defined for real $a>0$ and $-\pi<\beta<\pi$. Denote by $f(x ; a, \beta)$ the corresponding density, i.e.

$$
f(x ; a, \beta)=\frac{\left(2 \cos \frac{\beta}{2}\right)^{2 a}}{2 \pi \Gamma(2 a)}|\Gamma(a+i x)|^{2} e^{\beta x},
$$

and by $X$ the corresponding random variable.

Differentiating (4.13) with respect to $\beta$ and integrating the answer we get $\mathbb{E}(X)=a \tan \left(\frac{\beta}{2}\right)$ and $\operatorname{Var}(X)=\frac{1}{2} a \sec ^{2}\left(\frac{\beta}{2}\right)$. It is known that the corresponding Markov process has independent increments: the univariate law of $Y_{t}$ has density $f_{t}(x)=f(x ; A-t, \beta)$ and the transition densities are $f_{s, t}(y \mid x)=f(y-x ; t-s, \beta)$. One can verify also Chapman-Kolmogorov equations directly from the analog of Proposition 2.2 which is based on the identity

$$
\frac{f(y-x ; m, \beta) f(x ; a, \beta)}{f(y ; a+m, \beta)}=\varphi(x ; a, m-i y, a, m+i y) .
$$

The right hand side of (4.14) integrates to 1 because of (4.1). (This gives an "elementary" proof of the well known fact established by Laha and Lukacs [LL60, Lemma 2] that the hyperbolic secant laws form a convolution semi-group.)

The following proposition describes in more detail bridges mentioned in [BW09, Remark 4.1]. 
Proposition 4.3. All bridges of a hyperbolic secant process are described by formulas (4.6) and (4.7). Conversely, all quadratic harnesses with $0<\sigma \tau<1, \gamma=1-2 \sqrt{\sigma \tau}$ and $\eta, \theta \in \mathbb{R}$ such that $\eta \sqrt{\tau}+\theta \sqrt{\sigma}=0$ and $\theta^{2}<4 \tau$ can be realized as such bridges.

Proof. From (4.14) we see that for a hyperbolic secant process $\left(Y_{t}\right)$, the two-sided conditional law of $\mathcal{L}\left(Y_{t} \mid Y_{s}=x, Y_{u}=z\right)$ is given by

$$
g(y \mid x, z)=\varphi(y-x ; t-s, u-t-i(z-x), t-s, u-t+i(z-x)) .
$$

Inspecting formula (4.1), we see that

$$
\begin{gathered}
\varphi(y-x ; t-s, u-t-i(z-x), t-s, u-t+i(z-x))=\varphi(y ; t-s-i x, u-t-i z, t-s+i x, u-t+i z) \\
=\varphi(y ; u-t-i z, t-s-i x, u-t+i z, t-s+i x) .
\end{gathered}
$$

So identifying this with the univariate law of the bridge at time $S<t<U$, conditioned at $S<U$, we can read out that the bridge corresponds to the Markov process with transition probabilities (4.7), where $A=U-i Y_{U}, B=-S-i Y_{S}$.

\section{StANDARD BETA INTEGRAL}

In this section we use the well known beta density

$$
f(x ; a, b)=\frac{\Gamma(a+b)}{\Gamma(a) \Gamma(b)} x^{a-1}(1-x)^{b-1}
$$

to re-derive the quadratic harness properties of the one-parameter family of Dirichlet processes from [BW09, Eample 4.1]. (Here, the density is on $0<x<1$, and the parameters satisfy $a, b>0$.) It is well know that the corresponding random variable $X$ has moments

$$
\mathbb{E}(X)=\frac{a}{a+b}, \quad \text { and } \quad \operatorname{Var}(X)=\frac{a b}{(a+b)^{2}(a+b+1)} .
$$

The analog of (2.7) is the algebraic identity

$$
\frac{\frac{1}{1-x} f\left(\frac{y-x}{1-x} ; m, b\right) f(x ; a, b+m)}{f(y ; a+m, b)}=\frac{1}{y} f(x / y ; a, m) .
$$

In particular, we have a "convolution formula",

$$
\int_{0}^{y} \frac{1}{1-x} f\left(\frac{y-x}{1-x} ; m, b\right) f(x ; a, b+m) d x=f(y ; a+m, b) .
$$

Given $A>0$, we now use (5.4) to define the Markov process $\left(Y_{t}\right)_{0<t<A}$ by specifying its univariate laws as

$$
f_{t}(x)=f(x ; t, A-t), x \in[0,1],
$$

and for $s<t, y \geq x$ its transition probabilities as

$$
f_{s, t}(y \mid x)=\frac{1}{1-x} f\left(\frac{y-x}{1-x} ; t-s, A-t\right) .
$$

A calculation based on (5.4) shows that these expressions indeed satisfy the Chapman-Kolmogorov equations, so Markov process $\left(Y_{t}\right)_{t \in(0, A)}$ is well defined. (The same conclusion can be reached via probabilistic arguments, as these processes arise as bridges of the gamma process.)

From (5.2), $\mathbb{E}\left(Y_{t}\right)=t / A$ and $\operatorname{Var}\left(Y_{t}\right)=\frac{t(A-t)}{A^{2}(A+1)}$, and with some more work one can read out that $\operatorname{Cov}\left(Y_{s}, Y_{t}\right)=\frac{s(A-t)}{A^{2}(A+1)}$ for $s \leq t$. 
Since (5.3) shows that two-sided conditional laws are also beta, from (5.2) we can read out the conditional moments

$$
\begin{gathered}
\mathbb{E}\left(Y_{t}-Y_{s} \mid Y_{s}, Y_{u}\right)=\frac{t-s}{u-s}\left(Y_{u}-Y_{s}\right), \\
\operatorname{Var}\left(Y_{t} \mid Y_{s}, Y_{u}\right)=\frac{(t-s)(u-t)}{(u-s)^{2}((u-s)+1)}\left(Y_{u}-Y_{s}\right)^{2} .
\end{gathered}
$$

Applying Proposition 1.1 with $M=\frac{1}{A \sqrt{A+1}}, \beta=1 / A,=A, \epsilon \rightarrow-1$ (the remaining parameters are 0$)$, we see that $\left(Y_{t}\right)$ can be transformed into a quadratic harness on $T^{\prime}=(0, \infty)$ with parameters

$$
\eta=-\theta=-2 / \sqrt{1+A}, \sigma=\tau=1 /(1+A), \gamma=1-2 /(1+A)^{2} .
$$

(This is consistent with [BW09, Eample 4.1].)

Acknowledgement. We would like to thank Arthur Krener and Ofer Zeitouni for information on reciprocal processes, and to J. Wesołowski for several related discussions. This research was partially supported by NSF grant \#DMS-0904720, and by the Taft Research Center.

\section{REFERENCES}

[Ask89] R. Askey. Beta integrals and the associated orthogonal polynomials. Number Theory (ed. K. Alladi). Lecture Notes in Mathematics, 1395:84-121, 1989.

[BMW07] Włodzimierz Bryc, Wojciech Matysiak, and Jacek Wesołowski. Quadratic harnesses, q-commutations, and orthogonal martingale polynomials. Trans. Amer. Math. Soc., 359:5449-5483, 2007. arxiv.org/abs/math.PR/0504194.

[BW09] Włodek Bryc and Jacek Wesołowski. Conditioning of quadratic harnesses. http://arxiv.org/abs/0903.0150, 2009.

[BW10] Włodek Bryc and Jacek Wesołowski. Askey-Wilson polynomials, quadratic harnesses and martingales. Annals of Probability, 38:1221-1262, 2010. arxiv.org/abs/0812.0657.

[dB72] L. de Branges. Tensor product spaces. J. Math. Anal. Appl, 38:109-148, 1972.

[Jam74] Benton Jamison. Reciprocal processes. Z. Wahrscheinlichkeitstheorie und Verw. Gebiete, 30:65-86, 1974.

[JP88] Jean Jacod and Philip Protter. Time reversal on Lévy processes. Ann. Probab., 16(2):620-641, 1988.

[KS98] Roelof Koekoek and Rene F. Swarttouw. The Askey scheme of hypergeometric orthogonal polynomials and its $q$-analogue, 1998. Delft University of Technology Report no. 98-17, http://fa.its.tudelft.nl/ koekoek/askey.html.

[LL60] R. G. Laha and E. Lukacs. On a problem connected with quadratic regression. Biometrika, 47(300):335$343,1960$.

[Mei34] J. Meixner. Orthogonale polynomsysteme mit einer besonderen gestalt der erzeugenden funktion. $J$. London Math. Soc, 9(6), 1934.

[MY05] Roger Mansuy and Marc Yor. Harnesses, Lévy bridges and Monsieur Jourdain. Stochastic Process. Appl., 115(2):329-338, 2005.

[Wes93] Jacek Wesołowski. Stochastic processes with linear conditional expectation and quadratic conditional variance. Probab. Math. Statist., 14:33-44, 1993.

[Wil80] J.A. Wilson. Some hypergeometric orthogonal polynomials. SIAM Journal on Mathematical Analysis, 11:690, 1980.

Department of Mathematical Sciences, University of Cincinnati, PO Box 210025, Cincinnati, OH 45221-0025, USA 